\title{
Revisiting crash spatial heterogeneity: a Bayesian spatially varying coefficients approach
}

\author{
Pengpeng XU1, Helai HUANG²*, Ni DONG³, S.C. WONG1
}

${ }^{1}$ Department of Civil Engineering, The University of Hong Kong, Pokfulam Road, Hong Kong, China

2 School of Traffic \& Transportation Engineering, Central South University, Changsha, Hunan, China

${ }^{3}$ School of Transportation \& Logistics, Southwest Jiaotong University, Chengdu, Sichuan, China

*Correspondence: huanghelai@csu.edu.cn

\begin{abstract}
This study was performed to investigate the spatially varying relationships between crash frequency and related risk factors. A Bayesian spatially varying coefficients model was elaborately introduced as a methodological alternative to simultaneously account for the unstructured and spatially structured heterogeneity of the regression coefficients in predicting crash frequencies. The proposed method was appealing in that the parameters were modeled via a conditional autoregressive prior distribution, which involved a single set of random effects and a spatial correlation parameter with extreme values corresponding to pure unstructured or pure spatially correlated random effects.

A case study using a three-year crash dataset from the Hillsborough County, Florida, was conducted to illustrate the proposed model. Empirical analysis confirmed the presence of both unstructured and spatially correlated variations in the effects of contributory factors on severe crash occurrences. The findings also suggest that ignoring spatially structured heterogeneity may result in biased parameter estimates and incorrect inferences, while assuming the regression coefficients to be spatially clustered only is probably subject to the issue of over-smoothness.
\end{abstract}

Keywords: Crash frequency; spatial heterogeneity; unobserved heterogeneity; conditional autoregressive prior; Bayesian inference

\section{Introduction}

Modeling crash data involving contiguous spatial units, such as road networks and traffic analysis zones (TAZs), has gained growing research interests in the road traffic safety domain. This allows safety analysts to identify the clustering pattern of crashes, to better understand the factors that contribute to crash occurrences, and to recommend targeted countermeasures. Conventional crash prediction models, including the commonly used negative binomial and Poisson lognormal models, have an underlying assumption that their observations should be mutually independent. This fundamental requirement is almost always violated, because crash data collected in close proximity usually display spatial dependence (Quddus, 2008). The inclusion of spatially correlated effects typically has two main benefits. First, considering spatial correlation helps site estimates to pool strength from their neighbors, thereby improving model estimations (Aguero-Valverde and Jovanis, 2008). Second, spatial dependence can serve as a surrogate for unobserved covariates that vary smoothly over the region of interest (Cressie, 1993).

A range of spatial statistical techniques have been used to incorporate this spatial dependence into crash frequency modeling. The Bayesian hierarchical models are primarily used in these analyses, in which the spatial correlation is modeled via a set of random effects at the second level of hierarchy (Miaou et al., 2003; MacNab, 2004; Aguero-Valverde and Jovanis, 2006, 2008, 2010, 2014; Quddus, 2008; El-Basyouny and Sayed, 2009a; Mitra, 2009; Guo et al., 2010; Huang and Abdel-Aty, 2010; Siddiqui and Abdel-Aty, 2012; Flask and Schneider, 2013; Wang et al., 2013a; Xie et al., 2013; Dong et al., 2014, 2016; Xu et al., 2014; Zeng and 
Huang, 2014; Lee et al., 2015; Huang et al., 2016; Wang and Huang, 2016; Wang et al., 2016). This effect is mostly derived from the intrinsic conditional autoregressive (CAR) prior distribution proposed by Besag et al. (1991), which is a special case of Gaussian Markov random fields (Rue and Held, 2005). Alternative CAR specifications were also introduced by Richardson et al. (1992), Cressie (1993), and Leroux et al. (1999). Lee (2011) made a comprehensive comparison and concluded that the model of Leroux et al. (1999) was most appealing, as it performed consistently well in the presence of independence and strong spatial correlation.

Although most safety analysts have made an effort to handle the spatially correlated effects in model residuals, a limited number of studies have specifically focused on another issue related to the location dimension of crash data, i.e., spatial heterogeneity or spatial non-stationarity (Xu and Huang, 2015). Variables do not usually vary identically across space, and the relationship between crashes and related risk factors may not necessarily be constant or fixed across the study area. The possibility of accounting for this spatial heterogeneity by allowing some or all parameters to vary spatially holds considerable promise.

One possible method is the random parameters count-data models. Some of the many factors that influence crash occurrences are not observed or are nearly impossible to collect. If these unobserved factors were correlated with observed ones, biased parameters would be estimated and incorrect inference could be drawn (Mannering and Bhat, 2014). The random parameters approach has therefore been used to account for the unobserved heterogeneity in crash frequency (Anastasopoulos and Mannering, 2009; EI-Basyouny and Sayed, 2009b, 2011; Dinu and Veeraragavan, 2011; Ukkusuri et al., 2011; Venkataraman et al., 2013; Barua et al., $2015,2016)$. The regression coefficients in these random parameters models typically arise independently from some univariate distributions, and no attention is paid to the locations to which the parameters refer. This hypothesis may be inappropriate, particularly in cases where the unobserved factors are correlated over space (Xu and Huang, 2015). To capture this spatially structured variability in the effects of contributory factors, $\mathrm{Xu}$ and Huang (2015) advocated the development of a model based on the principle that the estimated parameters on a geographical surface are related to each other with closer values more similar than distant ones.

To address this potential spatial correlation in varying coefficients, two competing approaches are promising, i.e., the geographically weighted Poisson regression (GWPR; Fotheringham et al., 2002; Nakaya et al., 2005) and the Bayesian spatially varying coefficients (BSVC) models (Congdon, 1997; Assuncao et al., 2002; Congdon, 2003; Gelfand et al., 2003). The geographically weighted approach is one of the most innovative techniques in geography and has become increasingly prevalent in spatial econometrics, ecology analysis and disease mapping (Yao et al., 2015a). The method is similar in spirit to local linear models, relying on the calibration of multiple regression models for different geographical entities. Recently published studies have empirically demonstrated the superiority of the GWPR model with a substantial improvement in model goodness-of-fit and the ability to explore the spatially varying relationships between crash counts and predicting factors (Hadayeghi et al., 2010; Li et al., 2013; Pirdavani et al., 2014a, 2014b; Shariat-Mohaymany et al., 2015; Xu and Huang, 2015; Yao et al., 2015b).

Another potential method is the BSVC. The BSVC model has long been emerging in statistics as a methodological alternative to examine the non-constant linear relationships between variables (Congdon, 1997). The varying coefficients in the BSVC model can be selectively modeled as the geostatistical (Gelfand et al., 2003), intrinsic CAR (Congdon, 1997; Assuncao et al., 2002), or multiple membership processes (Congdon, 2003). Such an approach fits naturally into the Bayesian paradigm, where all parameters are treated as unknown random quantities. Obviously, the BSVC model differs from the GWPR in that the former is a single statistical model specified in a hierarchical manner, whereas the latter is an assembly of 
local spatial regression models, each fits separately. Wheeler and Calder (2007) conducted a series of simulation studies to evaluate the accuracy of regression coefficients in these two types of models under the presence of collinearity. Their evidence suggested that the BSVC model produced more accurate and more easily interpreted inferences, thus providing more flexibility (Wheeler and Calder, 2007). However, to assume the regression coefficients to be spatially clustered only is a strong prior belief. In reality, spatial pooling with smoothly varying coefficients over contiguous areas may be implausible, especially when clear discontinuities exist (Congdon, 2014, p. 340). In this vein, a robust model with a mechanism to accommodate the global and local smoothing collectively would be preferable.

This study intends to investigate the spatially varying relationships between crash frequency and relevant risk factors using a fully Bayesian approach. To simultaneously determine the strength of the unstructured and spatially structured variations in model regression coefficients, the CAR prior distribution derived from Leroux et al. (1999) is elaborately extended to the spatially varying coefficients framework. The proposed method is illustrated based on a case study with a comprehensive dataset from Hillsborough County, Florida.

\section{Methodology}

We begin this section with a quick review of the fixed coefficients model commonly used for modeling spatially correlated errors in crash prediction. We then move on to detail how this basic model can be readily generalized to estimate the varying regression coefficients within a fully Bayesian context.

Let $Y_{i}$ denote the observed number of crashes in location $i(i=1,2, \ldots, n), \operatorname{EV}_{\mathrm{i}}$ the exposure, and $X_{i k}$ the $k$ th $(k=1,2, \ldots, p)$ explanatory variable. On the basis of Huang and Abdel-Aty (2010), we have:

$Y_{i} \sim \operatorname{Poisson}\left(\lambda_{i}\right)$

$\ln \left(\lambda_{i}\right)=\beta_{1}+\beta_{2} \ln \left(\mathrm{EV}_{i}\right)+\sum_{k=3}^{p} \beta_{k} X_{i k}+u_{i}+s_{i}$

where $\lambda_{1}$ is the parameter of the Poisson model (i.e., the expected number of crashes in site $i) ; \quad \beta_{1}$ is the intercept; $\beta_{k}(k=2, \ldots, p)$ refers to the $k$ th regression coefficient to be estimated; $u_{i}$ denotes the pure unstructured effect, which could be specified via an exchangeable normal prior, i.e., $u_{i} \sim \mathrm{N}\left(0, \sigma_{u}^{2}\right)$; and $s_{i}$ is the spatially structured or spatially correlated error.

One widely used joint density for the spatial effects $\mathbf{s}=\left(s_{1}, s_{2}, \ldots, s_{n}\right)$ is in terms of pairwise differences in errors and a variance term $\sigma_{s}^{2}$ (Besag et al., 1991):

$$
P\left(s_{1}, s_{2}, \ldots, s_{n}\right) \propto \exp \left[-0.5\left(\sigma_{s}^{2}\right)^{-1} \sum_{i \sim j} c_{i j}\left(s_{i}-s_{j}\right)^{2}\right]
$$

This joint density implies a normal conditional prior for $s_{i}$ conditioning on the effect of $s_{j}$ in the remaining observations:

$s_{i} \mid s_{j \neq i} \sim \mathrm{N}\left(\frac{\sum_{j} c_{i j} s_{j}}{\sum_{j} c_{i j}}, \frac{\sigma_{s}^{2}}{\sum_{j} c_{i j}}\right)$

where $c_{i j}$ represents the non-normalized weight, e.g., $c_{i j}=1$ if $i$ directly connects with $j$, otherwise $c_{i j}=0$ (with $c_{i i}=0$ ); and $\sigma_{s}^{2}$ is the variance parameter, which controls the amount of extra variations due to spatial correlation. It is worth noting that this intrinsic CAR specification permits contiguity and distance-based weight matrices, but precludes the $k$ th- 
Although the univariate conditional prior distribution in equation (3) is well defined, the corresponding joint prior distribution for $\mathbf{s}$ is now improper (i.e., undefined mean and infinite variance; Sun et al., 1999). This fact probably leads to problems in convergence and identifiability in Bayesian estimation (Eberly and Carlin, 2000).

An alternative strategy to gain propriety is based on the strength of a single set of random effects $\mathbf{v}=\left(v_{1}, v_{2}, \ldots, v_{n}\right)$ :

$\ln \left(\lambda_{i}\right)=\beta_{1}+\beta_{2} \ln \left(\mathrm{EV}_{i}\right)+\sum_{k=3}^{p} \beta_{k} X_{i k}+v_{i}$ (1999):

Following Lee (2011), $v_{i}$ here is specified as the CAR prior proposed by Leroux et al.

$v_{i} \mid v_{j \neq i} \sim \mathrm{N}\left(\frac{\rho_{v} \sum_{j} c_{i j} v_{j}}{1-\rho_{v}+\rho_{v} \sum_{j} c_{i j}}, \frac{\sigma_{v}^{2}}{1-\rho_{v}+\rho_{v} \sum_{j} c_{i j}}\right)$

where $\rho_{v}\left(0 \leq \rho_{v} \leq 1\right)$ is the spatial correlation parameter, with $\rho_{v}=0$ simplifying to an independent identically distributed normal prior, and an increase in its value toward one indicating an increasing spatial correlation. Accordingly, setting $\rho_{v}=1$ corresponds to the improper CAR as in equation (3).

Based on the factorization theorem, $\mathbf{v}=\left(v_{1}, v_{2}, \ldots, v_{n}\right)$ results in a joint multivariate Gaussian distribution (Congdon, 2008):

$\mathbf{v} \sim \operatorname{MVN}\left(\mathbf{0}, \sigma_{v}^{2}\left[\rho_{v} \mathbf{K}+\left(1-\rho_{v}\right) \mathbf{I}\right]^{-1}\right)$

where $\mathbf{I}$ is an $n \times n$ identity matrix, and the elements of $\mathbf{K}$ are calculated as:

$K_{i j}= \begin{cases}\sum_{j} c_{i j} & \text { if } i=j \\ -c_{i j} & \text { if } i \neq j\end{cases}$

Despite the local relationship is incorporated through the covariance structure of the error term, the outputs from the preceding models still consist of a set of global parameter estimates. Intuitively, the local variations can be addressed by setting the regression slopes as random effects ${ }^{1}$, allowing the effects of covariates to vary spatially:

$\ln \left(\lambda_{i}\right)=\beta_{1}+\beta_{i 2} \ln \left(\mathrm{EV}_{i}\right)+\sum_{k=3}^{p} \beta_{i k} X_{i k}+v_{i}$

where $\beta_{i k}$ is the coefficient of the $k$ th explanatory variable for site $i$. In practice, one may assume $\beta_{i k}$ as an independent normal distribution (i.e., $\mathrm{N}\left(\mu_{k}, \sigma_{k}^{2}\right)$ ) in accordance with EI-Basyouny and Sayed (2009) and Barua et al. (2015), or alternatively as a pure spatially correlated effects as illustrated by Assuncao et al. (2002), Congdon (2003), and Gelfand et al. (2003). However, the variations in the regression coefficients are very likely to arise from both unstructured and spatially structured effects. On this occasion, we have:

\footnotetext{
${ }^{1}$ In fully Bayesian analysis, if the priors relates to random effects, the specification involves the form of distribution and the naming of its parameters, followed by the assignment of values to these parameters in a higher stage prior. By contrast, the prior for a fixed effect involves just one stage of specification.
} 
$\boldsymbol{\beta}_{\mathbf{k}} \sim \operatorname{MVN}\left(\boldsymbol{\mu}_{\mathbf{k}}, \sigma_{k}^{2}\left[\rho_{k} \mathbf{K}+\left(1-\rho_{k}\right) \mathbf{I}\right]^{-1}\right)$

Unlike equation (6), the formula in equation (9) has a constant non-zero mean $\boldsymbol{\mu}_{\mathbf{k}}=\left(\mu_{k}, \ldots, \mu_{k}\right)$, in which $\mu_{k}$ is the overall estimate of the regression slope, denoting the average of the posterior estimates of $\boldsymbol{\beta}_{\mathbf{k}}\left(\beta_{1 k}, \beta_{2 k}, \ldots, \beta_{n k}\right)$. The precision matrix is now given by $\rho_{k} \mathbf{K}+\left(1-\rho_{k}\right) \mathbf{I}$, which is a weighted average of spatially correlated and independent structures (denoted as $\mathbf{K}$ and $\mathbf{I}$, respectively). This specification is capable of accounting for a range of weak and strong spatial correlations in regression coefficients, with $\rho_{k}=0$ decreasing to the spatially independent random effects and an increase in $\rho_{k}$ to the value of one representing spatial smoothing only.

The univariate full conditional distribution corresponding to equation (9) is given as follows:

$\beta_{i k} \mid \beta_{j k} \sim \mathrm{N}\left(\frac{\rho_{k} \sum_{j} c_{i j} \beta_{j k}+\left(1-\rho_{k}\right) \mu_{k}}{1-\rho_{k}+\rho_{k} \sum_{j} c_{i j}}, \frac{\sigma_{k}^{2}}{1-\rho_{k}+\rho_{k} \sum_{j} c_{i j}}\right)$

Specifically, the conditional expectation of $\beta_{i k}$ is a weighted average of the random effects at neighboring sites and the overall mean $\mu_{k}$, and the conditional variance has a compelling methodological interpretation. When the regression coefficients present a strong spatial correlation, $\rho_{k}$ would be close to one and the conditional variance approaches $\sigma_{k}^{2} / \sum_{j} c_{i j}$. This variance configuration recognizes that in the presence of a strong spatial correlation, the more neighbors a site has, the more information in the data about the value of its random effects. In comparison, if the random effect is spatially independent, the conditional variance becomes $\sigma_{k}^{2}$. Apparently, the parameter $\rho_{k}\left(0 \leq \rho_{k} \leq 1\right)$ can serve as an indicator to assess the relative strength of spatial and unstructured variations in the estimated coefficients. Besides, if there is no significant heterogeneity in $\boldsymbol{\beta}_{\mathbf{k}}$, the $\sigma_{k}^{2}$ then displays a dispersion with the mean of its posterior distribution lower than the standard deviation (Barua et al., 2015). In this case, the regression slopes are better fitted as the fixed effects.

Obtaining the fully Bayesian posterior estimates requires the specification of prior distributions. Prior distributions are typically used to reflect prior knowledge about the parameters of interest. If such prior information is available, it would be encouraged to formulate the so-called informative priors (Yu and Abdel-Aty, 2013; Heydari et al., 2014). In the absence of sufficient prior knowledge, non-informative (i.e., vague) prior could be applied to model parameters:

$\beta_{k} \sim \mathrm{N}(0,1000)$

$\mu_{k} \sim \mathrm{N}(0,1000)$

In light of a study by Congdon (2008), the spatial correlation parameters $\rho_{v}$ and $\rho_{k}$ were assigned as a uniform $(0,1)$. Given that the commonly used inverse-Gamma $(\varepsilon, \varepsilon)$ priors (where $\varepsilon$ is a small number, e.g., 0.01 or 0.001 ) are sensitive to the value of $\varepsilon$ if the true variance is close to zero, a uniform $(0,10)$ was finally specified for $\sigma_{v}$ and $\sigma_{k}$, respectively (Gelman, 2006).

For model comparison and selection, three commonly used measures, i.e., $R_{d}^{2}$, mean absolute deviance (MAD), and Deviance Information Criterion (DIC) were employed. 


$$
R_{d}^{2}=1-\frac{\sum_{i=1}^{n}\left(Y_{i}-\hat{\lambda}_{i}\right)^{2} / \hat{\lambda}_{i}}{\sum_{i=1}^{n}\left(Y_{i}-\bar{Y}\right)^{2} / \bar{Y}}
$$

where $\hat{\lambda}_{i}$ denotes the expected crash number obtained by the crash prediction models, and $\bar{Y}$ is the average of crash frequency. The model with $R_{d}^{2}$ towards value of one fits better to the data.

The MAD was adopted to provide a measure of model prediction performance:

$$
\mathrm{MAD}=\frac{1}{n} \sum_{i=1}^{n}\left|\hat{\lambda}_{i}-Y_{i}\right|
$$

A smaller value of MAD suggests that on average the model predicts the observed crash data better.

Meanwhile, the penalized goodness of fit measure, i.e., DIC was also used here to take model complexity into account:

$\mathrm{DIC}=D(\bar{\theta})+2 p_{D}=\bar{D}+p_{D}$

where $D(\bar{\theta})$ is the deviance evaluated at $\bar{\theta}$, the posterior means of the parameters; $p_{D}$ is the effective number of parameters in the model; and $\bar{D}$ is the posterior mean of the deviance statistic $D(\bar{\theta})$. The lower the DIC, the better the model fit. In General, differences in DIC of more than 10 definitely rule out the model with the higher DIC, differences between 5 and 10 are considered substantial, and a difference of less than 5 indicates that the models are not statistically different (Spiegelhalter et al., 2002).

\section{Data preparation}

To illustrate the application of the proposed BSVC models, a case study was conducted based on a dataset from Hillsborough County, Florida. A total of 57,694 crashes were recorded from the year 2005 to 2007. Of these, 4854 (8.41\%) were reported as severe crashes with fatalities and severe injuries. Road and traffic-related factors were mainly extracted from the Florida Department of Transportation's roadway characteristics inventory and geographical information maps for Hillsborough. These variables included the daily vehicle miles traveled (DVMT), trip productions and attractions, intersections, and road segment lengths with various speed limits. A number of factors reflecting the demographic and socioeconomic features were also downloaded from the United States Census reports.

Hillsborough contains $738 \mathrm{TAZs}$ in total. The shape file of TAZs was collected from the Florida Department of Transportation District 7's Intermodal Systems Development Unit. To assign the boundary crashes, a buffer zone with the size of $100 \mathrm{ft}$ (i.e., 30.48 meters) was created around the TAZ boundaries. Crashes located within the boundary buffer were then allocated to adjacent TAZs equally. This half-to-half ratio assignment method was recommended by Wei and Lovegrove (2010) and Washington et al. (2010). Other variables were also spatially attached to the respective TAZs in a similar way.

The variables available for model development, in addition to their descriptive statistics, are shown in Table 1. In this study, we selected the number of severe crashes as the dependent 
variable. The DVMT along with trips and total population was treated as the measures of exposure, as the model with multiple exposure variables outperformed its counterpart using a single specification (Pridavani et al., 2012; Lee et al., 2015). The explanatory variables were those commonly used in previous macroscopic safety analyses (Aguero-Valverde and Jovanis, 2006; Quddus, 2008; Hadayeghi et al., 2010; Huang et al., 2010; Pridavani et al., 2012; Li et al., 2013; Lee et al., 2014, 2015). Concerning the spatial weight matric, as a default option, the adjacency-based first-order neighbors (i.e., $c_{i j}=1$ if and only if $\mathrm{TAZ}_{i}$ shared a common boundary with $\mathrm{TAZ}_{j}$ ) were considered here for convenience. This neighborhood structure was also widely employed in current macroscopic crash analysis (Aguero-Valverde and Jovanis, 2006; Quddus, 2008; Huang et al., 2010; Siddiqui and Abdel-Aty, 2012; Wang et al., 2013; Lee et al., 2014; Xu et al., 2015; Dong et al., 2016).

12 Table 1. Summary of variables and descriptive statistics.

\begin{tabular}{|c|c|c|c|c|c|}
\hline Variables & Definition & Mean & SD & Min & Max \\
\hline \multicolumn{6}{|c|}{ Predictor Variable } \\
\hline Severe crashes & Total number of fatal and severe injury crashes per TAZ & 6.58 & 7.02 & 0.00 & 47.00 \\
\hline \multicolumn{6}{|c|}{ Exposure Variables } \\
\hline DVMT & Daily vehicle miles traveled (in thousands) & 95.07 & 110.24 & 0.06 & 788.77 \\
\hline TRIP & Trip production and attraction (in thousands) & 10.46 & 9.12 & 0.09 & 108.36 \\
\hline POP & Total population (in thousands) & 1.31 & 1.27 & 0.00 & 9.48 \\
\hline \multicolumn{6}{|c|}{ Explanatory Variables } \\
\hline Inter_density & Number of intersections/road length & 3.17 & 5.61 & 1.00 & 66.12 \\
\hline Road density & $\begin{array}{l}\text { Total road segment length/area (miles per acre, in } \\
\text { hundreds) }\end{array}$ & 2.07 & 1.14 & 0.00 & 7.44 \\
\hline Seglen15 & Percent of road segment length with 15 -mph speed limit & 2.27 & 4.98 & 0.00 & 52.52 \\
\hline Seglen 25 & Percent of road segment length with 25 -mph speed limit & 72.01 & 20.80 & 0.00 & 100.00 \\
\hline Seglen35 & Percent of road segment length with $35-\mathrm{mph}$ speed limit & 17.73 & 15.36 & 0.00 & 100.00 \\
\hline Seglen45 & Percent of road segment length with $45-\mathrm{mph}$ speed limit & 2.10 & 5.32 & 0.00 & 43.78 \\
\hline Seglen55_65 & $\begin{array}{l}\text { Percent of road segment length with } 55-\text { to } 65-\mathrm{mph} \text { speed } \\
\text { limit }\end{array}$ & 5.10 & 10.47 & 0.00 & 83.27 \\
\hline Male & Proportion of male population & 49.98 & 9.96 & 0.00 & 100.00 \\
\hline POP_15 & Proportion of population below 15 years of age & 21.23 & 7.77 & 0.00 & 43.25 \\
\hline POP_65 & Proportion of population above 65 years of age & 12.67 & 11.77 & 0.00 & 100.00 \\
\hline MHINC & Median household income (USD, in thousands) & 40.14 & 20.24 & 0.00 & 115.66 \\
\hline WORKERS & Percent of workers & 43.94 & 14.58 & 0.00 & 90.91 \\
\hline WT_PRV & Percent of workers taking motor vehicles to work & 87.10 & 19.11 & 0.00 & 100.00 \\
\hline WT_PUB & Percent of workers taking public transportation to work & 1.96 & 3.71 & 0.00 & 27.27 \\
\hline WT_BIC & Percent of workers taking bicycles to work & 0.70 & 1.56 & 0.00 & 12.90 \\
\hline WT_WALK & Percent of workers walking to work & 2.17 & 3.62 & 0.00 & 40.00 \\
\hline WT_HOME & Percent of workers working at home & 2.62 & 2.83 & 0.00 & 23.08 \\
\hline
\end{tabular}

\section{Results and discussions}

The proposed models were estimated in a fully Bayesian context using Markov chain Monte Carlo simulation. The freeware software WinBUGS (Spiegelhalter et al., 2005) was used to calibrate the models. Two parallel chains with diverse starting values were tracked. The first 10,000 iterations in each chain were discarded as burn-ins. 5000 iterations were then performed for each chain, resulting in a sample distribution of 10,000 for each parameter. The model's convergence was monitored by the Brooks-Gelman-Rubin statistic, visual examination 
1 of the Markov chain Monte Carlo chains, and the ratios of Monte Carlo errors relative to the 2 respective standard deviations of the estimates. As a rule of thumb, these ratios should be less 3 than 0.05 . 
For model specification, a correlation test was first conducted to ensure the non-inclusion of highly correlated variables. The correlation analysis indicated a high correlation between the percent of road segment length with a speed limit of $25 \mathrm{mph}$ and the percent of road segment length with a speed limit of $35 \mathrm{mph}$, as the value of Pearson product-moment correlation coefficient for these two variables was equal to 0.79 . This result implied that those two variables should not be included together in the model. The DIC was then used to compare alternative models with different covariate subsets. The one producing a lower DIC value was considered superior.

For comparison purpose, in addition to the proposed BSVC model, we considered three candidate models in which the regression coefficients were modeled as fixed effects, unstructured random effects, and pure spatially correlated random effects, respectively. As such, four models were eventually estimated. In this section, the performance of these models is compared, followed by the parameter estimates presented and discussed.

\subsection{Model performance comparison}

Table 2 shows the results of the goodness-of-fit measures for the calibrated models. The regression coefficients in these four models were respectively specified as the $N(0,1000)$, $\mathrm{N}\left(\mu_{k}, \sigma_{k}^{2}\right)$, intrinsic CAR prior of Besag et al. (1991), and CAR prior of Leroux et al. (1999). The results indicated that the consideration of spatial heterogeneity could considerably improve model performance. In particular, the developed BSVC-3 model performed best with the highest $R_{d}^{2}$ as well as the lowest MAD and DIC values. This finding suggested that the cross-sectional variability in crash counts could be better explained if the unstructured and spatially correlated variations in regression coefficients were simultaneously addressed. Besides, the BSVC-1 model was found to be comparable with the fixed coefficients counterpart in terms of model goodness of fit. Chen and Tarko (2014) reported a similar conclusion when using the random parameters and random effects models (the intercept was randomly distributed with the regression coefficients fixed) to analyze work zone safety.

Table 2 Measures of model goodness-of-fit

\begin{tabular}{llccc}
\hline Model & Regression coefficients structure & $R_{d}^{2}$ & MAD & DIC \\
\hline Basic & Fixed effects & 0.79 & 2.58 & 3535.92 \\
BSVC-1 & Unstructured random effects & 0.79 & 2.57 & 3534.83 \\
BSVC-2 & Pure spatially correlated random effects & 0.82 & 2.46 & 3527.86 \\
BSVC-3 & Unstructured and spatially correlated random effects & $\mathbf{0 . 8 4}$ & $\mathbf{2 . 3 8}$ & $\mathbf{3 5 2 2 . 8 7}$ \\
\hline
\end{tabular}

\subsection{Parameters estimates}

Table 3 summarizes the parameter estimates in the basic and spatially varying coefficients models. A $5 \%$ level of significance was used as the threshold to determine whether the parameters differed from zero. Any variables that were insignificant in all four models were excluded. As shown in Table 3, the following factors were associated with a significant positive relationship with severe crash counts: DMVT, number of trips, population, and the percentage of road segments with a $45-\mathrm{mph}$ speed limit. Affluent TAZs with a higher percentage of road segments with a speed limit of $25 \mathrm{mph}$ and the greater use of bicycles by workers tend to be relatively safer in terms of severe crash rates. In addition, the median household income consistently resulted in a significant variation in the coefficient (i.e., the posterior mean of $\hat{\sigma}_{\text {MHINC }}^{2}$ was higher than its standard deviation). 
Table 3. Estimates results for the basic and spatially varying coefficients models.

\begin{tabular}{|c|c|c|c|c|c|c|c|c|}
\hline & \multicolumn{2}{|l|}{ Basic } & \multicolumn{2}{|l|}{ BSVC-1 } & \multicolumn{2}{|l|}{ BSVC-2 } & \multicolumn{2}{|l|}{ BSVC-3 } \\
\hline & Mean(SD) & $95 \%$ BCI & Mean(SD) & $95 \% \mathrm{BCI}$ & Mean(SD) & $95 \% \mathrm{BCI}$ & Mean(SD) & $95 \%$ BCI \\
\hline Intercept & $1.427(0.062)^{* *}$ & $(1.304,1.550)$ & $1.425(0.070)^{* *}$ & $(1.282,1.564)$ & $1.425(0.076)^{* *}$ & $(1.268,1.569)$ & $1.430(0.069)^{* *}$ & $(1.290,1.563)$ \\
\hline $\ln (\mathrm{DVMT})$ & $0.549(0.036)^{* *}$ & $(0.479,0.620)$ & $0.540(0.036)^{* *}$ & $(0.470,0.612)$ & $0.548(0.038)^{* *}$ & $(0.474,0.623)$ & $0.544(0.036)^{* *}$ & $(0.473,0.616)$ \\
\hline $\ln (\mathrm{TRIP})$ & $0.128(0.035)^{* *}$ & $(0.059,0.197)$ & $0.121(0.036)^{* *}$ & $(0.051,0.191)$ & $0.126(0.036)^{* *}$ & $(0.056,0.197)$ & $0.122(0.036)^{* *}$ & $(0.052,0.192)$ \\
\hline $\ln (\mathrm{POP})$ & $0.290(0.053)^{* *}$ & $(0.187,0.392)$ & $0.324(0.056)^{* *}$ & $(0.216,0.434)$ & $0.298(0.058)^{* *}$ & $(0.183,0.410)$ & $0.324(0.056)^{* *}$ & $(0.214,0.435)$ \\
\hline Seglen25 & $-0.099(0.041)^{* *}$ & $(-0.177,-0.019)$ & $-0.109(0.041)^{* *}$ & $(-0.189,-0.029)$ & $-0.103(0.042)^{* *}$ & $(-0.184,-0.021)$ & $-0.110(0.041)^{* *}$ & $(-0.190,-0.030)$ \\
\hline Seglen45 & $0.065(0.030)^{* *}$ & $(0.005,0.124)$ & $0.069(0.030)^{* *}$ & $(0.010,0.127)$ & $0.067(0.030)^{* *}$ & $(0.008,0.126)$ & $0.068(0.030)^{* *}$ & $(0.009,0.126)$ \\
\hline MHINC & $-0.127(0.044)^{* *}$ & $(-0.212,-0.041)$ & $-0.156(0.048)^{* *}$ & $(-0.251,-0.062)$ & $-0.155(0.051)^{* *}$ & $(-0.254,-0.055)$ & $-0.157(0.056)^{* *}$ & $(-0.268,-0.048)$ \\
\hline WT_BIC & $-0.074(0.033)^{* *}$ & $(-0.139,-0.010)$ & $-0.059(0.035)^{*}$ & $(-0.128,0.009)$ & $-0.060(0.035)^{*}$ & $(-0.130,0.009)$ & $-0.060(0.035)^{*}$ & $(-0.129,0.009)$ \\
\hline$\hat{\sigma}_{\text {MHINC }}^{2}$ & $\longrightarrow$ & $\longrightarrow$ & $0.077(0.033)^{* *}$ & $(0.012,0.146)$ & $0.190(0.082)^{* *}$ & $(0.056,0.377)$ & $0.194(0.112)^{* *}$ & $(0.043,0.463)$ \\
\hline$\hat{\sigma}_{v}^{2}$ & $0.944(0.162)^{* *}$ & $(0.674,1.302)$ & $0.820(0.145)^{* *}$ & $(0.570,1.133)$ & $0.807(0.157)^{* *}$ & $(0.569,1.196)$ & $0.799(0.145)^{* *}$ & $(0.549,1.113)$ \\
\hline$\hat{\rho}_{\text {MHINC }}$ & $\longrightarrow$ & $\square$ & 0 & $\longrightarrow$ & 1 & $\longrightarrow$ & $0.390(0.286)^{* *}$ & $(0.020,0.913)$ \\
\hline$\hat{\rho}_{v}$ & $0.588(0.133)^{* *}$ & $(0.363,0.883)$ & $0.706(0.145)^{* *}$ & $(0.432,0.975)$ & $0.647(0.152)^{* *}$ & $(0.372,0.958)$ & $0.684(0.149)^{* *}$ & $(0.407,0.968)$ \\
\hline
\end{tabular}

Note: SD refers to the standard deviation. BCI refers to the Bayesian confidence interval. ${ }^{* *}$ and ${ }^{*}$ indicate $5 \%$ and $10 \%$ levels of significance, respectively. 
Several general observations are worth mentioning. First, unlike the basic model whose coefficients were restricted to be constant, the BSVC models allowed the regression coefficients to vary spatially. Hence, one crash prediction model was applied for the entire area using the basic model, whereas by virtue of BSVC, different crash prediction models could be estimated for individual TAZ. Second, the significant variables were not entirely identical between the fixed and BSVC models. For example, the percentage of residents who took bicycles to work appeared to be less significant in the BSVC models. This inconsistency was likely due to model misspecification, including the neglect of spatial heterogeneity. Third, it is interesting to observe that the error variability (i.e., $\hat{\sigma}_{v}^{2}$ ) obviously decreased, dropping from 0.944 to approximately 0.80 when variations were introduced in the regression coefficients. This was expected to some extent, as the heterogeneity in the regression slopes could capture some of the extra variations previously explained by the random effects in error term. More importantly, although the average estimate of the median household income (i.e., $\hat{u}_{\text {мнім }}$ ) was fairly similar across the three BSVC models, the spatial correlation parameter $\hat{\rho}_{\text {MHINC }}$ in model BSVC-3 produced a posterior estimate with a mean of 0.390 and a standard deviation of 0.286 , implying that a moderate proportion of variations (around $40 \%$ ) was explained by the spatially correlated effects. The corresponding 95\% Bayesian confidence interval was reported as $(0.020,0.913)$, which significantly differed from both zero and one. This finding demonstrated the presence of both unstructured and spatially structured variations in the effects of related risk factors in crash prediction.

To illustrate the distinctions in inference among the three BSVC models, an in-depth investigation into the estimates of the varying coefficients is believed to provide additional insights. The parameters of median household income generated for each TAZ (i.e., $\hat{\beta}_{\text {iMHINC }}$ ) are therefore plotted in Fig. 1, and their spatial patterns are further explored.

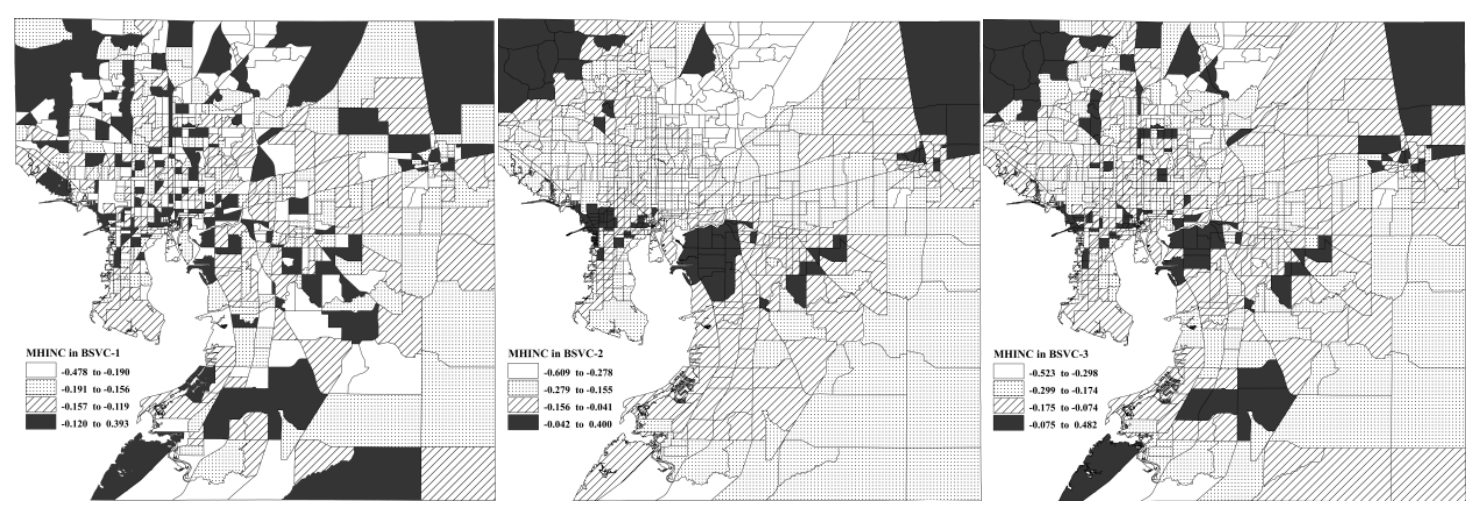

Fig. 1. Estimated parameters of median household income in the three BSVC models.

As shown in Fig. 1, the estimated parameters derived from the developed BSVC models revealed obvious spatial variations, but the three models produced notably different sets of results. Specifically, the mapped pattern of the BSVC-1 coefficients was apparently less smooth than that of the other two BSVC counterparts. This result was not surprising given that the BSVC-1 model made no spatial assumptions, allowing more noise to introduce roughness into the local parameter estimates. In contrast, the BSVC-2 model provided estimates using a mechanism essentially based on spatial smoothing. However, to assume the varying coefficients to be spatially clustered uniquely is prone to sustaining the risk of over-smoothness. In fact, heterogeneity in the effects of the explanatory variables may also occur due to the unstructured effects, analogous to white noise in time series. From this point of view, the proposed BSVC-3 model seemed more rational, as it not only allowed for a spatial pooling of strength when appropriate, but also adopted a strategy to reflect parameters that were discordant with those of surrounding areas. To illustrate this, Fig. 1 identified the overall pattern of the regression coefficients in the BSVC-3 model as spatial clustering, while the 
parameters for a small number of TAZs located in the northwest and south were visibly isolated from their neighbors.

To quantify the slope of spatial correlation in local coefficient estimates, Moran's I statistics were calculated and results are presented in Table 4 . As expected, the parameters in BSVC-2 and BSVC-3 models exhibited statistically significant spatial clustering (i.e., positive spatial correlation) at the $95 \%$ confidence level. Counterintuitively, a significant negative spatial correlation (i.e., spatial dispersion) was observed in the varying coefficients of BSVC-1. Note that these coefficients in model BSVC-1 were assumed to be spatially random distributed. This underlying model hypothesis was violated, and biased parameters might thus be produced.

Table 4. Moran's I statistic for the coefficients of median household incomes.

\begin{tabular}{lccc}
\hline Moran's I & $\boldsymbol{I}$ & Z score & $p$-value \\
\hline BSVC-1 & $-\mathbf{0 . 0 8 2 ^ { * * }}$ & $-\mathbf{2 . 0 2 9}$ & $\mathbf{0 . 0 4 2}$ \\
BSVC-2 & $0.411^{* *}$ & 10.307 & 0.000 \\
BSVC-3 & $0.094^{* *}$ & 2.378 & 0.017 \\
\hline
\end{tabular}

Note: ${ }^{* *}$ represents a $5 \%$ level of significance.

Given that the BSVC-3 model outperformed the other models, we use it to interpret our results. A good interpretation of the parameter estimates also helped to partially justify the validity of the developed model. According to Table 3, six variables finally produced statistically significant parameters with $95 \%$ BCIs bounded away from zero in BSCV-3: DVMT, number of trips, population, the percent of road segments with speed limits of 25 and $45 \mathrm{mph}$, and median household income. The percentage of workers who took bicycles to work was found significant at a $90 \%$ confidence level. The signs of these parameters were generally consistent with empirical judgments and previous studies.

DVMT, trips and population were included as exposure variables in the model. The coefficients were all significantly positive, implying that more severe crashes were expected in zones with higher concentrations of traffic volumes, travel demands, and residents. Similar results were also previously reported (Huang et al., 2010; Pridavani et al., 2012; Lee et al., 2015).

Looking at roadways with different speed limits, the percentage of road segments with a speed limit of $25 \mathrm{mph}$ was observed to have a significant negative relationship with severe crash frequency, while increasing the proportion of road segments with a speed limit of 45 mph was expected to lead to more fatal and severe injury crashes. This finding was consistent with the well-accepted fact that, with other risk factors held constant, higher speed is associated with more serious crash outcomes (Aarts and Schagen, 2006; Wang et al., 2013b).

Area deprivation level is supposed to be closely correlated with safety awareness, driving behavior, and transport facility conditions, and thus has an indirect influence on safety outcomes. In this study, the median household income resulted in a spatially varying coefficient with a posterior mean of -0.157 and a variance parameter of 0.194 . The magnitude of this coefficient ranged from -0.523 to 0.482 . Given these distributional parameters, $94.58 \%$ of the distribution indicated a negative effect on severe crash occurrence. An inspection of the BCIs implied that the majority of the TAZs with positive signs were insignificant. This result confirmed the results of most prior studies that deprived areas were more likely to suffer from higher casualty rates (Quddus, 2008; Huang et al., 2010; Lee et al., 2015).

At present, people are being encouraged to cycle more as a viable alternative and economical mode of transportation. Interestingly, the percentage of workers who took bicycles to work was reported to have a negative relationship with severe crashes at the $10 \%$ significance level. One potential explanation is that bicyclists typically have a strong value preference for "perceived" safe routes with lower speeds, lower traffic volumes, and well-designed infrastructural facilities (Jacobsen et al., 2009). As a result, areas in which more residents ride bicycles tend to be inherently safer. It is also noteworthy that perceived safety 
did not necessarily correspond with actual safety (Cho et al., 2009). Perceived safety without actual safety creates a false sense of security, while actual safety without perceived safety discourages people from bicycling. Therefore, to promote cycling, both the safety of facilities and the number of bicyclists should be increased.

\section{Conclusions}

Traffic crashes are complex events that involve dynamic interactions between traffic participants, vehicles, road geometric features, and environmental conditions. Given these complex circumstances, it seems impossible to access all of the data that potentially determine the likelihood of a crash. To deal with this challenge, random parameters models have been employed to address the unobserved heterogeneity, i.e., variations in the effects of variables across a sample population that are unknown to analysts (Mannering et al., 2016).

This study particularly focused on the spatial heterogeneity in crash prediction. The spatial heterogeneity here could be defined as "the continuous space-varying structural relationships describing space-related factors that systematically vary across the region of interest". We provided new insights to current research that in addition to unstructured variability, the heterogeneity in the effects of explanatory variables could also arise from the spatially correlated effects. For this purpose, an alternative fully Bayesian approach was introduced to simultaneously accommodate the unstructured and spatial structured variations in model parameters. The proposed method was superior in the sense that the regression coefficients were modeled via a single set of random effects and a spatial correlation parameter with extreme values corresponding to pure unstructured or pure spatially correlated random effects.

Based on a three-year crash dataset from the Hillsborough County, Florida, empirical analysis demonstrated the presence of both unstructured and spatially structured variations in the effects of contributory factors in severe crash occurrences. The results also suggested that ignoring spatially structured heterogeneity may result in biased estimates and incorrect inferences, while assuming the regression coefficients to be spatially clustered only is probably subject to the issue of over-smoothness.

Since crash data are typically collected in spatial proximity, we expect the present study to promote awareness of the spatial dimension of crashes among safety analysts, i.e., the discrimination between "analysis of spatial data" and "spatial data analysis". Despite both types of studies involve data with geographical co-ordinates, the former effectively ignores the geographical component and treats data as if they were aspatial, while the latter makes use of the geographical component to explore the spatial aspects of the data.

For future research, apart from the typically used CAR model, other spatial prior distributions such as the jointly specified (Mitra, 2009; Aguero-Valverde, 2014) and multiple membership (El-Basyouny and Sayed, 2009a) forms could be attempted. Considering that the model calibrated in our study is applicable to a univariate cross-sectional outcome, further efforts to extend the approach to multivariate and longitudinal dimensions are also highly advocated. Furthermore, sicne the results of the study are based on a single dataset, future studies with different data sources would prove worthwhile to enhance our findings.

\section{Acknowledgements}

This work was jointly supported by the Joint Research Scheme of National Natural Science Foundation of China/Research Grants Council of Hong Kong (Project No. 71561167001 \& N_HKU707/15), the Natural Science Foundation of China (Project No. 71371192), and the Research Fund for the Fok Ying Tong Education Foundation of Hong Kong (Project No. 142005). We would like to sincerely thank Dr. Mohamed Abdel-Aty at the University of Central Florida and the Florida Department of Transportation for providing the data. 


\section{References}

Aarts, L., Schagen, I.V., 2006. Driving speed and the risk of road crashes: a review. Acci. Anal. Prev. 38, 215-224.

Aguero-Valverde, J., Jovanis, P.P., 2006. Spatial analysis of fatal and injury crashes in Pennsylvania. Acci. Anal. Prev. 38, 618-625.

Aguero-Valverde, J., Jovanis, P.P., 2008. Analysis of road crash frequency with spatial correlation. Transp. Res. Rec. 2061, 55-63.

Aguero-Valverde, J., Jovanis P.P., 2010. Spatial correlation in multilevel crash frequency models: effects of different neighboring structures. Transp. Res. Rec. 2165, 21-32.

Aguero-Valverde, J., 2014. Direct spatial correlation in crash frequency models: estimation of the effective range. J. Transport. Saf. Sec. 6(1), 21-33.

Anastasopoulos, P.Ch., Mannering, F.L., 2009. A note on modeling vehicle-accident frequencies with random parameter count models. Acci. Anal. Prev. 41(1), 153-159.

Assuncao, R.M., Potter, J.E., Cavenaghi, S.M., 2002. A Bayesian space varying parameter model applied to estimating fertility schedules. Stat. Med. 21, 2057-2075.

Barua, S., El-Basyouny, K., Islam, M.T., 2015. Effects of spatial correlation in random parameter collision count-data models. Anal. Methods Accid. Res. 5-6, 28-42.

Barua, S., El-Basyouny, K., Islam, M.T., 2016. Multivariate random parameters collision count data models with spatial heterogeneity. Anal. Methods Accid. Res. 9, 1-15.

Besag, J., York, J., Molli, E.A., 1991. Bayesian image restoration with two applications in spatial statistics. Annu. Inst. Stat. Math. 43(1), 1-59.

Chen, E., Tarko, A.P., 2014. Modeling safety of highway work zones with random parameters and random effects models. Anal. Methods Accid. Res. 1, 86-95.

Cho, G., Rodriguez, D., Khattak, A., 2009. The role of the built environment in explaining relationships between perceived and actual pedestrian and bicyclist safety. Acci. Anal. Prev. 41(4), 692-702.

Congdon, P., 1997. Bayesian models for spatial incidence: a case study of suicide using the BUGS program. Health \& Place 3(4), 229-247.

Congdon, P., 2003. Modelling spatially varying impacts of socioeconomic predictors on mortality outcomes. J. Geogr. Syst. 5, 161-184.

Congdon, P., 2008. A spatially adaptive conditional autoregressive prior for area health data. Statist. Med. $5,552-563$.

Congdon, P., 2014. Applied Bayesian Modelling (Second Edition). John Wiley \& Sons, Chichester.

Cressie, N., 1993. Statistics for spatial data. John Wiley \& Sons, New York.

Dinu, R.R., Veeraragavan, A., 2011. Random parameter models for accident prediction on two-lane undivided highways in India. J. Saf. Res. 42(1), 39-42.

Dong, N., Huang, H., Xu, P., Ding, Z., Wang, D., 2014. Evaluating spatial-proximity structures in crash prediction models at the level of traffic analysis zones. Transp. Res. Rec. 2432, 46-52.

Dong, N., Huang, H., Lee, J., Gao, M., Abdel-Aty, M., 2016. Macroscopic hotspots identification: a Bayesian spatio-temporal interaction approach. Acci. Anal. Prev. 92, 256-264.

Eberly, L., Carlin, B., 2000. Identifiability and convergence issues for Markov chain Monte Carlo fitting of spatial models. Stat. Med. 19, 2279-2294.

El-Basyouny, K., Sayed, T., 2009a. Urban arterial accident prediction models with spatial effects. Transp. Res. Rec. 2102, 27-33.

El-Basyouny, K., Sayed, T., 2009b. Accident prediction models with random corridor parameters. Acci. Anal. Prev. 41(5), 1118-1123.

EI-Basyouny, K., Sayed, T., 2011. A full Bayes multivariate intervention model with random parameters among matched pairs for before-after safety evaluation. Acci. Anal. Prev. 43, 87-94.

Flask, T., Schneider, W., 2013. A Bayesian analysis of multi-level spatial correlation in single vehicle motorcycle crashes in Ohio. Saf. Sci. 53, 1-10.

Fotheringham, A.S., Brunsdon, C., Charlton, M.E., 2002. Geographically Weighted Regression: The Analysis of Spatially Varying Relationship. Wiley, Chichester. 
Gelfand, A.E., Kim, H-J., Sirmans, C.F., Banerjee, S., 2003. Spatial modeling with spatially varying coefficient processes. J. Amer. Statist. Assoc. 98, 387-396.

Gelman, A., 2006. Prior distributions for variance parameters in hierarchical models. Bayesian Anal. 1, 515-533.

Guo, F., Wang, X., Abdel-Aty, M.A., 2010. Modeling signalized intersection safety with corridor-level spatial correlations. Acci. Anal. Prev. 42, 84-92.

Hadayeghi, A., Shalaby, A.S., Persaud, B.N., 2010. Development of planning level transportation safety tools using geographically weighted Poisson regression. Acci. Anal. Prev. 42(2), 676-688.

Heydari, S., Miranda-Moreno, L.F., Lord, M., Fu, L., 2014. Bayesian methodology to estimate and update safety performance functions under limited data conditions: a sensitivity analysis. Acci. Anal. Prev. 64, 41-51.

Huang H., Abdel-Aty M., 2010. Multilevel data and Bayesian analysis in traffic safety. Acci. Anal. Prev. 42(6), 1556-1565.

Huang, H., Abdel-Aty, M., Darwiche, A.L., 2010. County-level crash risk analysis in Florida: Bayesian spatial modeling. Transp. Res. Rec. 2148, 27-37.

Huang, H., Song, B., Xu, P., Zeng, Q., Lee, J., Abdel-Aty, M., 2016. Macro and micro models for zonal crash prediction with application in hot zones identification. J. Tran. Geogr. 54, 248-256.

Jacobsen, P.L., Racioppi, F., Rutter, H., 2009. Who owns the roads? How motorized traffic discourages walking and bicycling. Inj. Prev. 15, 369-373.

Lee, D., 2011. A comparison of conditional autoregressive models used in Bayesian disease mapping. Spat. Spatio-temporal Epidemiol. 2, 79-89.

Lee, J., Abdel-Aty, M., Choi, K., 2014. Analysis of residence characteristics of at-fault drivers in traffic crashes. Saf. Sci. 68, 6-13.

Lee, J., Abdel-Aty, M., Choi, K., Huang, H., 2015. Multi-level hot zone identification for pedestrian safety. Acci. Anal. Prev. 76, 64-73.

Leroux, B., Lei, X., Breslow, N., 1999. Estimation of disease rates in small areas: a new mixed model for spatial dependence. In Halloran, M., Berry, D., (eds), Statistical Models in Epidemiology, the Environment and Clinical Trials. New York: Springer-Verlag, 135-178.

Li, Z., Wang, W., Liu, P., Bigham, J.M., Ragland, D.R., 2013. Using geographically weighted Poisson regression for county-level crash modeling in California. Saf. Sci. 58, 89-97.

MacNab, Y.C., 2004. Bayesian spatial and ecological models for small-area accident and injury analysis. Acci. Anal. Prev. 36, 1019-1028.

Mannering, F.L., Bhat, C.R., 2014. Analytic methods in accident research: methodological frontier and future directions. Anal. Methods Accid. Res. 1, 1-22.

Mannering, F.L., Shankar, V., Bhat, C.R., 2016. Unobserved heterogeneity and the statistical analysis of highway accident data. Anal. Methods Accid. Res. 11, 1-16.

Miaou, S., Song, J.J., Mallick, B.K., 2003. Roadway traffic crash mapping: a space-time modeling approach. J. Tranp. Stat. 6(1), 33-57.

Mitra, S., 2009. Spatial autocorrelation and Bayesian spatial statistical method for analyzing intersections prone to injury crashes. Transp. Res. Rec. 2136, 92-100.

Nakaya, T., Fotheringham, A.S., Brunsdon, C., Charlton, M., 2005. Geographically weighted Poisson regression for disease association mapping. Stat. Med. 24, 2695-2717.

Pirdavani, A., Brijis, T., Bellemans, T., Kochan, B., Wets, G., 2012. Application of different exposure measures in development of planning-level zonal crash prediction models. Transp. Res. Rec. 2280, 145-153.

Pirdavani, A., Bellemans, T., Brijs, T., Kochan, B., Wets, G., 2014a. Assessing the road safety impacts of a teleworking policy by means of geographically weighted regression method. J. Tran. Geogr. 39, 96-110.

Pirdavani, A., Bellemans, T., Brijs, T., Wets, G., 2014b. Application of geographically weighted regression technique in spatial analysis of fatal and injury crashes. J. Transp. Eng. 140(8), 04014032.

Quddus, M.A., 2008. Modeling area-wide count outcomes with spatial correlation and heterogeneity: an analysis of London crash data. Acci. Anal. Prev. 40(4), 1486-1497. 
Richardson, S., Guihenneuc, C., Lasserre, V., 1992. Spatial linear models with autocorrelated error structure. The Statistician 41, 539-557.

Rue, H., Held, L., 2005. Gaussian Markov Random Fields: Theory and Applications. Chapman \& Hall/CRC.

Siddiqui, C., Abdel-Aty, M., 2012. Nature of modeling boundary pedestrian crashes at zone. Transport. Res. Rec. 2299, 31-40.

Shariat-Mohaymany, A., Shahri, M., Mirbagheri, B., Matkan, A.A., 2015. Exploring spatial non-stationarity and varying relationships between crash data and related factors using geographically weighted Poisson regression. Transactions in GIS 19(2), 321-337.

Spiegelhalter, D.J., Best, N.G., Carlin, B.P., Van Der Linde, A., 2002. Bayesian measures of model complexity and fit. J. R. Stat. Soc. Ser. B 64(4), 583-639.

Spiegelhalter, D., Thomas, A., Best, N., Lunn, D., 2005. WinBUGS user manual. MRC Biostatistics Unit, Cambridge, United Kingdom.

Sun, D., Tsutakawa, R., Speckman, P.L., 1999. Bayesian inference for CAR(1) models with noninformative priors. Biometrika 86, 341-350.

Ukkusuri, S., Hasan, S., Aziz, H.M.A., 2011. Random parameter model used to explain effects of built-environment characteristics on pedestrian crash frequency. Transp. Res. Rec. 2237, 98-106.

Venkataraman, N., Ulfarsson, G.F., Shankar, V.N., 2013. Random parameter models of interstate crash frequencies by severity number of vehicles involved, collision and location type. Acci. Anal. Prev. 59, 309-318.

Wang, J., Huang, H., 2016. Road network safety evaluation using Bayesian hierarchical joint model. Acci. Anal. Prev. 90, 152-158.

Wang, X., Wu, X., Abdel-Aty, M., Tremont, P.J., 2013a. Investigation of road network features and safety performance. Acci. Anal. Prev. 56, 22-31.

Wang, C., Quddus, M.A., Ison, S.G., 2013b. The effect of traffic and road characteristics on road safety: a review and future research direction. Saf. Sci. 57, 264-275.

Wang, X., Yang, J., Lee, C., Ji, Z., You, S., 2016. Macro-level safety analysis of pedestrian crashes in Shanghai, China. Accid. Anal. Prev. 96, 12-21.

Washington, S.P., Van Schalkwyk, I., You, D., Shin, K., Samuelson, J.P., 2010. Forecasting the safety impacts of socio-demographic changes and safety countermeasures. NCHRP 8-44(2): PLANSAFE User Manual. Transportation Research Board, Washington, DC.

Wei, F., Lovegrove, L., 2010. Boundary Effects in Developing Macro-level CPMs: a Case Study of City of Ottawa University of British Columbia (accessed 06.08.13.). Http: //www. cite7org/schol arships_awards/documents/2010_StudentPaper_FengWei.pdf.

Wheeler, D.C., Calder, C.A., 2007. An assessment of coefficient accuracy in linear regression models with spatially varying coefficients. J. Geogr. Syst. 9(2), 145-166.

Xie, K., Wang, X., Huang, H., Chen, X., 2013. Corridor-level signalized intersection safety analysis in Shanghai, China using Bayesian hierarchical models. Acci. Anal. Prev. 50, 25-33.

Xu, P., Huang, H., Dong, N., Abdel-Aty, M., 2014. Sensitivity analysis in the context of regional safety modeling: identifying and assessing the modifiable areal unit problem. Acci. Anal. Prev. 70, 110-120.

$\mathrm{Xu}$, P., Huang, H., 2015. Modeling crash spatial heterogeneity: random parameter versus geographically weighting. Acci. Anal. Prev. 75, 16-25.

Yao, S., Loo, B.P.Y., Yang, B.Z., 2015a. Traffic collisions in space: four decades of advancement in applied GIS. Ann. GIS 22(1), 1-14.

Yao, S., Loo, B.Y.P., Lam, W.W.Y., 2015b. Measures of activity-based pedestrian exposure to the risk of vehicle-pedestrian collisions: space-time path vs. potential path tree methods. Acci. Anal. Prev. 75, 320-332.

Yu, R., Abdel-Aty, M., 2013. Investigating different approaches to develop informative priors in hierarchical Bayesian safety performance functions. Acci. Anal. Prev. 75, 16-25.

Zeng, Q., Huang, H., 2014. Bayesian spatial joint modeling of traffic crashes on an urban road network. Acci. Anal. Prev. 67, 105-112. 